\title{
Kane's Equations for Nonholonomic Systems in Bond-Graph-Compatible Velocity and Momentum Forms
}

\author{
James R. Phillips ${ }^{1}$, Farid Amirouche ${ }^{2}$ \\ ${ }^{1}$ Senior Applications Engineer \\ 2 Department of Orthopaedic Surgery \\ Applied Dynamics International \\ University of Illinois at Chicago \\ Ann Arbor, MI, USA \\ phillips@adi.com \\ Chicago, IL, USA \\ amirouch@uic.edu
}

\begin{abstract}
The authors' previously published results on a nonholonomic momentum form of Kane's equations are extended from scleronomic to rheonomic systems. The momentum form is found to be partially Hamiltonian, and a new velocity form is found that is partially Lagrangian. The results are derived for general particle systems, and then specialized to rigid-body systems. Except for externally constrained velocities, all system matrices are independent of time, making the results power-conserving, and suitable for use with bond graphs. A new nonholonomic IC (NIC) bond-graph element is defined, and bond graphs for the new results are exhibited using this element.
\end{abstract}

Keywords: Kane's equations, bond graph, NIC element, partially Hamiltonian, partially Lagrangian.

\section{INTRODUCTION}

Bond graphs are a widely used graphical formalism for representing dynamic systems, which may encompass multiple energy domains, in a uniform fashion, using a small set of ideal elements $[1,2]$. Prior to the appearance of our 2018 paper [3], the most advanced methods for representing multibody systems in a concise bond-graph form were based on generalized momentum, using the IC-field bond-graph element, and these were limited to holonomic systems. In [3] we introduced a bond-graph-compatible momentum method for nonholonomic systems, based on Kane's equations $[4,5,6]$, but it was limited to scleronomic systems. In this paper we further extend our momentum method to incorporate systems with external time-varying constraints, and we find that this momentum method is partially Hamiltonian (to be defined below). We also introduce a velocity-based method that is partially Lagrangian (also to be defined below). Finally, we introduce a generalization of the IC bond-graph element, the nonholonomic, or NIC bond-graph element, and exhibit bond graphs for our nonholonomic methods.

Kane's formulation is used in the development, because it can provide concise, matrix-based descriptions of multibody systems [7]. The methodology here differs from [7] however, in that it makes no assumptions about the kinematic formulation, other than that a set of partial velocity vectors describing the system is available. For the greatest generality, we begin with particle systems, and then specialize the results to systems of rigid bodies.

\section{SCOPE, ASSUMPTIONS AND APPROACH}

\subsection{Degrees of freedom, generalized coordinates, generalized velocities}

We consider a simple nonholonomic system $[6,8]$ in an inertial frame, with all rheonomic constraints initially relaxed, making it scleronomic. Therefore assume $R$ generalized coordinates $q_{r}$ completely determine the positions of all particles in the frame, and $S$ generalized velocities $f_{S}$ completely determine the coordinate derivatives $\dot{q}_{r} ; S$ is the number of scleronomic degrees of freedom for the system, and the number of nonholonomic constraints is $R-S$. The coordinate derivatives and generalized velocities are related through the matrix equation

$$
\dot{\mathbf{q}}=\mathbf{Q f},
$$


where $\mathbf{q}$ and $\dot{\mathbf{q}}$ are length $R$ column matrices of coordinates and coordinate derivatives, $\mathbf{f}$ is a length $S$ column matrix of generalized velocities, and $\mathbf{Q}$ is an $R \times S$ matrix of rank $S$, a function only of the generalized coordinates q. After finding equations of motion for the scleronomic degrees of freedom $\mathbf{f}$, we will then apply $S_{\mathrm{c}}$ rheonomic constraints, so that the final number of unconstrained degrees of freedom for the system is $S-S_{\mathrm{c}}$. Without loss of generality, we assume the rheonomic constraints are directly known functions of time. Thus when the rheonomic constraints are applied, we will partition the $\mathbf{f}$ matrix as

$$
\mathbf{f}=\left[\begin{array}{l}
{ }^{u} \mathbf{f} \\
{ }^{{ }} \mathbf{f}
\end{array}\right]
$$

where ${ }^{\mathrm{c}} \mathbf{f}$ is a length $S_{\mathrm{c}}$ column matrix of known constrained generalized velocities, and ${ }^{\mathrm{u}} \mathbf{f}$ is a length $S-S_{\mathrm{c}}$ column matrix of unconstrained generalized velocities.

Remark. Although the precise technical meaning of holonomicity is that $S=R$, we shall describe the system equations of motion as being in holonomic form only when $\mathbf{f}=\dot{\mathbf{q}}$. This is the case for classical dynamics methodologies such as Lagrange's and Hamilton's equations. The introduction of the $\mathbf{Q}$ matrix allows the use of so-called nonholonomic velocities as elements of the generalized velocity vector $\mathbf{f}$, which for multibody systems is a vital convenience in formulating the equations of motion. Selection of generalized velocities $f_{s}$ to minimize the complexity of the equations of motion is treated in $[9,10]$.

\subsection{Particle velocities and partial velocities}

By assumption, the velocity $v$ of every particle in the system can be expressed as

$$
\boldsymbol{v}=\left(\frac{\partial \boldsymbol{v}}{\partial \mathbf{f}^{\top}}\right) \mathbf{f},
$$

where $\partial \boldsymbol{v} / \partial \mathbf{f}^{\top}$ is a row matrix of partial derivatives, each of which is a function only of the generalized coordinates q. These are nonholonomic partial velocities, in Kane's terminology [6, 11]. The particle notation will be extended to model rigid bodies in Section 4.

For the purpose of formulating correct equations of motion, we require knowledge of these partial velocity vectors. Any competent method for formulating the system kinematics could be used for this purpose, e.g. the use of lower-body arrays [7]; however, we need not account here for the lower-level details of the kinematic formulation.

\section{DEVELOPMENT OF GENERAL PARTICLE-BASED FORMS OF THE EQUATIONS}

\subsection{Impressed and Constraint Forces}

We assume that every particle, having differential mass $\mathrm{d} m$, is subject to a differential force resultant $\mathrm{d} \boldsymbol{R}$, given by

$$
\mathrm{d} \boldsymbol{R}=\mathrm{d} \boldsymbol{F}+\mathrm{d}^{\mathrm{c}} \boldsymbol{F},
$$

where $\mathrm{d} \boldsymbol{F}$ is the total impressed force, and $\mathrm{d}^{\mathrm{c}} \boldsymbol{F}$ is the total constraint force. The constraint force $\mathrm{d}^{\mathrm{c}} \boldsymbol{F}$ is that force, and only that force, required to enforce the motion constraints implied by the partial velocity vectors defined above. The impressed force $\mathrm{d} \boldsymbol{F}$ represents all other forces acting on the particle, which may be specified by constitutive laws, or may be unspecified functions of time. All dissipative or friction effects are accounted for in the impressed forces, as well as all conservative or otherwise unspecified actuation forces not considered to be constraint reactions.

Remark. This is standard analytical dynamics terminology for impressed and constraint forces, as expounded in e.g. [12]. However Kane's terminology for $\mathrm{d} \boldsymbol{F}$, as used in [6], is "active force", while his preferred terminology for $\mathrm{d}^{\mathrm{c}} \boldsymbol{F}$ is "noncontributing force". In [11], which is a revised and updated edition of [6], both "noncontributing force" and "constraint force" are used to describe $\mathrm{d}^{\mathrm{c}} \boldsymbol{F}$. 


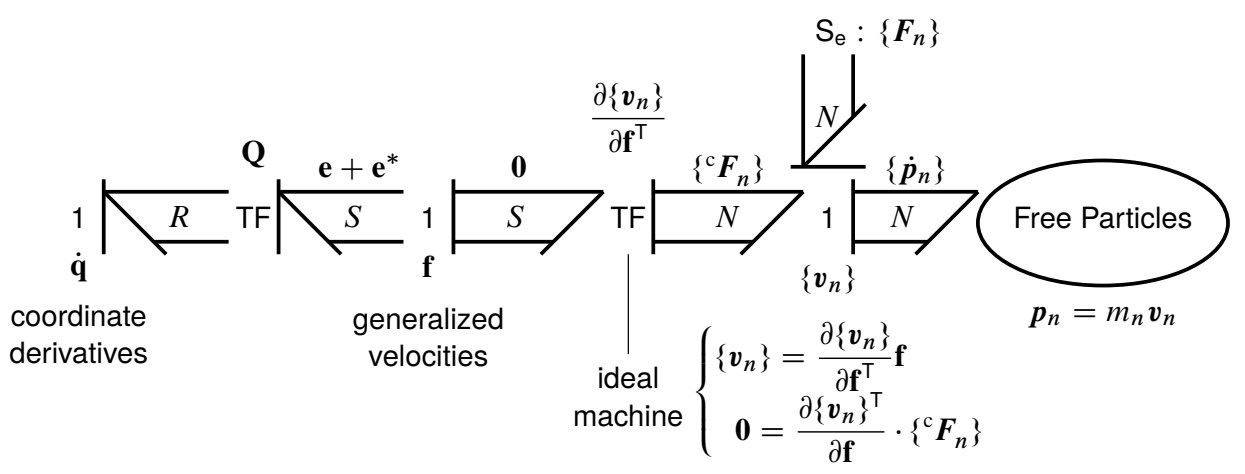

Figure 1. Multibond graph of unreduced Kane's equations

\subsection{Kane's Equations in Unreduced Form}

Defining the differential linear momentum of each particle as

$$
\mathrm{d} \boldsymbol{p}=\boldsymbol{v} \mathrm{d} m,
$$

we may write Newton's second law as

$$
\mathrm{d} \dot{\boldsymbol{p}}=\mathrm{d} \boldsymbol{R} .
$$

Kane's equations in unreduced form for the particle system are (using Stieltjes integration over the particle system):

$$
\int\left(\frac{\partial \boldsymbol{v}}{\partial \mathbf{f}}\right) \cdot(\mathrm{d} \dot{\boldsymbol{p}}-\mathrm{d} \boldsymbol{F})=\mathbf{0},
$$

where $\mathbf{0}$ is a length $S$ column matrix of zeros; in view of (4) this is equivalent to saying

$$
\int\left(\frac{\partial \boldsymbol{v}}{\partial \mathbf{f}}\right) \cdot\left(\mathrm{d}^{\mathrm{c}} \boldsymbol{F}\right)=\mathbf{0} .
$$

Eq. (8) is a consequence of the principle of virtual power, which is a generalization of the more well known principle of virtual work, that is applicable to nonholonomic systems [13].

We define the total generalized impressed (or "active") force matrix $\mathbf{e}$ as

$$
\mathbf{e} \equiv \int\left(\frac{\partial \boldsymbol{v}}{\partial \mathbf{f}}\right) \cdot \mathrm{d} \boldsymbol{F}
$$

so that we have

$$
\int\left(\frac{\partial \boldsymbol{v}}{\partial \mathbf{f}}\right) \cdot \mathrm{d} \dot{\boldsymbol{p}}=\mathbf{e}
$$

as the unreduced form of Kane's equations for a particle system.

Remark. Practitioners of Kane's methodology typically write (10) as

$$
\mathbf{e}+\mathbf{e}^{*}=\mathbf{0},
$$

where the elements of the $\mathbf{e}^{*}$ column matrix are called "generalized inertia forces", defined as

$$
\mathbf{e}^{*} \equiv-\int\left(\frac{\partial \boldsymbol{v}}{\partial \mathbf{f}}\right) \cdot \mathrm{d} \dot{\boldsymbol{p}} .
$$

Equation (11) is an homage to D'Alembert [4]. 


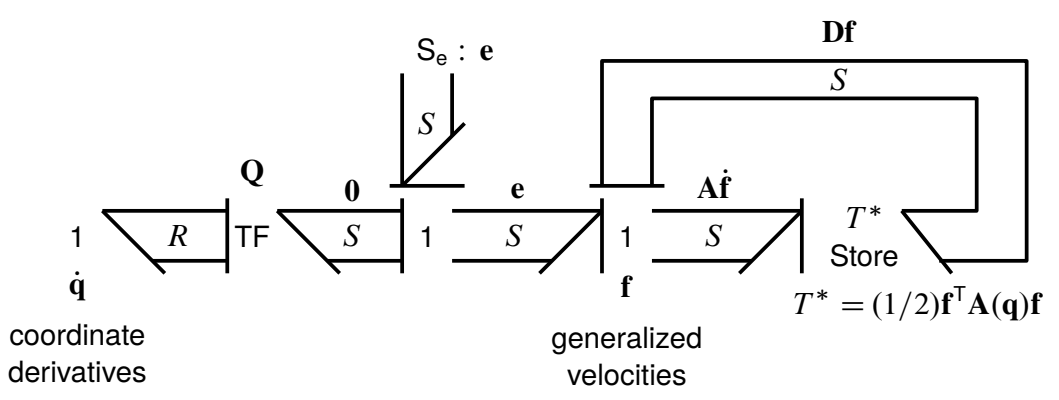

Figure 2. Multibond graph of reduced Kane's equations, velocity form

\subsection{Bond Graph Representation of the Unreduced Equations}

Figure 1 displays a multibond graph [14, 15] of Kane's equations in unreduced form. Here we have converted from a Stieltjes integral representation of differential particle masses to a large but finite sum of $N$ mass particles with inertial velocities $\boldsymbol{v}_{n}$ and masses $m_{n}$. There are three 1 -junctions (common flow, effort summing) on the bond graph, with associated flow matrices $\dot{\mathbf{q}}, \mathbf{f}$, and $\left\{\boldsymbol{v}_{n}\right\}$ from left to right. There are two transformer (TF) elements connecting the three 1-junctions; one to represent the transformation of the $\mathbf{Q}$ matrix, and the other to represent the transformation of the partial velocity vectors.

The constitutive equations for the multibond TF element with modulus given by the partial velocity vectors are written below it. They imply perfect power conservation by this TF element (all bond graph TF elements have this property). Essentially the principle of virtual power represents the constraint forces in such a way that, in combination with the partial velocity vectors, they form an ideal machine, represented by the TF element.

The inertial subsystem on the right for the free particles is in so-called derivative causality, as indicated by the position of the causal stroke on the multibond, and its associated efforts are transferred across the TF element to the left side of the $\mathbf{f} 1$-junction as $\mathbf{e}^{*}$. Similarly the impressed forces transfer to the same location as e. Meanwhile there is a zero matrix on the right side of that 1 -junction, because there are no effort sources to the left of that 1-junction. The sum of efforts on the left side of the $\mathbf{f} 1$-junction yields the unreduced form of Kane's equations as expressed in (11). This could be considered a bond-graphic derivation of Kane's equations, because the bond-graph representation of the system as an ideal machine is evidently equivalent to the principle of virtual power.

\subsection{Reduced Velocity Form and System Matrices}

To find the reduced velocity form of Kane's equations, we return to the representation of the system as an infinite collection of particles, each having an associated differential mass and momentum. Using the partial velocity expansion for $v$ from (3), we may write the derivative of the momentum vector for a particle as

$$
\mathrm{d} \dot{\boldsymbol{p}}=\left[\left(\frac{\partial \boldsymbol{v}}{\partial \mathbf{f}^{\top}}\right) \mathrm{d} m\right] \dot{\mathbf{f}}+\left[\frac{\mathrm{d}}{\mathrm{d} t}\left(\frac{\partial \boldsymbol{v}}{\partial \mathbf{f}^{\top}}\right) \mathrm{d} m\right] \mathbf{f} .
$$

Substituting this into the Stieltjes integral in (10), we find

$$
\mathbf{A} \dot{\mathbf{f}}+\overline{\mathbf{D}} \mathbf{f}=\mathbf{e},
$$

where $\mathbf{A}$ is the system mass matrix given by

$$
\mathbf{A} \equiv \int\left(\frac{\partial \boldsymbol{v}}{\partial \mathbf{f}}\right) \cdot\left(\frac{\partial \boldsymbol{v}}{\partial \mathbf{f}^{\top}}\right) \mathrm{d} m
$$


and $\overline{\mathbf{D}}$ is the system gyrator matrix given by

$$
\overline{\mathbf{D}} \equiv \int\left(\frac{\partial \boldsymbol{v}}{\partial \mathbf{f}}\right) \cdot \frac{\mathrm{d}}{\mathrm{d} t}\left(\frac{\partial \boldsymbol{v}}{\partial \mathbf{f}^{\top}}\right) \mathrm{d} m
$$

"gyrator" is standard bond-graph terminology for a matrix $\overline{\mathbf{D}}$ which maps a column matrix of input flows $\mathbf{f}$ to a column matrix of output efforts $\overline{\mathbf{D}}$.

$\mathbf{A}$ is a function only of $\mathbf{q}$, and by differentiation of (15), we find the identity

$$
\dot{\mathbf{A}}=\overline{\mathbf{D}}+\overline{\mathbf{D}}^{\top} \text {. }
$$

We will make use of this fact below. Although (16) provides a unique definition for $\overline{\mathbf{D}}$, any matrix $\mathbf{D}$ that satisfies (17) and for which $\mathbf{D f}=\overline{\mathbf{D}} \mathbf{f}$ will serve for $\overline{\mathbf{D}}$ in (14). These two properties also guarantee that $\mathbf{D}^{\top} \mathbf{f}=\overline{\mathbf{D}}^{\top} \mathbf{f}$. Equation (14) (with $\overline{\mathbf{D}}$ replaced by $\mathbf{D}$ ) is what we consider the standard reduced velocity form of Kane's equations, for scleronomic systems. It is applicable to systems composed of point masses, rigid bodies, and flexible bodies modeled with a finite number of degrees of freedom.

Remark. Flexible bodies will not be considered here, but in Sec. 4 we provide the formulas to compute $\mathbf{A}$ and $\mathbf{D}$ from rigid body mass properties and velocities. Clearly it is $\mathbf{D} \mathbf{f}$ rather than $\mathbf{D}$ that is actually required in (14), and in Sec. 3.7 below we also develop an alternative approach to computing $\mathbf{D f}$ as a whole rather than $\mathbf{D}$ separately from $\mathbf{f}$. One must be cautious in the interpretation of the Df term, because due to differentiation of the partial velocity vectors in (16), D is itself already linear in $\mathbf{f}$, so that the term as a whole is quadratic rather than linear in $\mathbf{f}$.

To develop a bond graph for the reduced velocity form of Kane's equations, we first consider the total kinetic co-energy function $T^{*}(\mathbf{q}, \mathbf{f})$ for the particle system, which can be written in terms of the mass matrix $\mathbf{A}$ as

$$
T^{*}(\mathbf{q}, \mathbf{f}) \equiv(1 / 2) \mathbf{f}^{\top} \mathbf{A}(\mathbf{q}) \mathbf{f} .
$$

The total rate of change of kinetic energy is then given as

$$
\begin{aligned}
\frac{\mathrm{d} T^{*}}{\mathrm{~d} t} & =\frac{\partial T^{*}}{\partial \mathbf{f}^{\top}} \dot{\mathbf{f}}+\frac{\partial T^{*}}{\partial \mathbf{q}^{\top}} \dot{\mathbf{q}} \\
& =\mathbf{f}^{\top} \mathbf{A} \dot{\mathbf{f}}+(1 / 2) \mathbf{f}^{\top} \dot{\mathbf{A}} \mathbf{f} \\
& =\mathbf{f}^{\top}(\mathbf{A} \dot{\mathbf{f}})+\mathbf{f}^{\top}(\mathbf{D} \mathbf{f}) .
\end{aligned}
$$

This suggests that a system kinetic co-energy store may be represented as having two multibond ports: one with effort Af , and one with effort $\mathbf{D f}$. A full multibond graph of the reduced form of the equations, using a two-port kinetic co-energy $\left(T^{*}\right)$ store, is shown in Fig. 2, where Eq. (14) is represented at the $\mathbf{f} 1$-junction to the left of the $T^{*}$ store. The left (I) port of the store, with effort Af , has flow causality, while the right (C) port of the store, with effort Df, has effort causality.

\subsection{Generalized Momentum Form and Generalized Gyration Forces}

Now consider the generalized momentum matrix $\mathbf{p}$, which we initially define as

$$
\mathbf{p} \equiv \int\left(\frac{\partial \boldsymbol{v}}{\partial \mathbf{f}}\right) \cdot d \boldsymbol{p}
$$

Using the definition of the mass matrix in (15), we can also write this as

$$
\mathbf{p}=\mathbf{A f} .
$$

Regarding $\mathbf{p}$ in (21) as a function $\hat{\mathbf{p}}(\mathbf{q}, \mathbf{f})$, we see that we could compute the mass matrix from the partial derivative of $\hat{\mathbf{p}}$ with respect to $\mathbf{f}$ :

$$
\hat{\mathbf{p}}(\mathbf{q}, \mathbf{f}) \equiv \mathbf{A}(\mathbf{q}) \mathbf{f},
$$


and therefore $\mathbf{A}$ is given by

$$
\mathbf{A}=\frac{\partial \hat{\mathbf{p}}}{\partial \mathbf{f}^{\top}}
$$

To find a differential equation for $\mathbf{p}$, differentiate (21) with respect to time, yielding

$$
\dot{\mathbf{p}}=\mathbf{A} \dot{\mathbf{f}}+\dot{\mathbf{A}} \mathbf{f} .
$$

Using Eq. (17) for $\dot{\mathbf{A}}$, and the velocity form (14) to eliminate $\mathbf{A} \dot{\mathbf{f}}$, we find the differential equation for $\mathbf{p}$ as:

$$
\dot{\mathbf{p}}=\mathbf{e}+\mathbf{D}^{\top} \mathbf{f} .
$$

This we regard as one of a pair of equations of motion. In order to integrate these equations for $\mathbf{p}$, we have to solve simultaneously for $\mathbf{f}$ from $\mathbf{p}$. Using (21), we find the second of the pair as

$$
\mathbf{f}=\mathbf{A}^{-1} \mathbf{p}
$$

Equations (25) and (26), taken as a pair, we consider to be Kane's momentum equations of motion for a scleronomic system.

The transpose of the gyrator matrix $\mathbf{D}$ is evidently itself a gyrator matrix, mapping the flow matrix $\mathbf{f}$ to an effort matrix $\mathbf{D}^{\top} \mathbf{f}$. Again, we should treat this product with care, because it is quadratic rather than linear in the generalized velocities $\mathbf{f}$. We name the output effort matrix $\hat{\mathbf{e}}$, and call it generalized gyration force:

$$
\hat{\mathbf{e}} \equiv \mathbf{D}^{\top} \mathbf{f}
$$

which allows us to write (25) as

$$
\dot{\mathbf{p}}=\mathbf{e}+\hat{\mathbf{e}} .
$$

The product $\mathbf{D}^{\top} \mathbf{f}=\overline{\mathbf{D}}^{\top} \mathbf{f}$ has a straightforward per-particle interpretation. Using the definition of $\overline{\mathbf{D}}$ from (16), we find

$$
\hat{\mathbf{e}}=\int \frac{\mathrm{d}}{\mathrm{d} t}\left(\frac{\partial \boldsymbol{v}}{\partial \mathbf{f}}\right) \cdot \mathrm{d} \boldsymbol{p},
$$

which allows us to define the differential generalized gyration force contributed by each particle as

$$
\mathrm{d} \hat{\mathbf{e}}=\frac{\mathrm{d}}{\mathrm{d} t}\left(\frac{\partial \boldsymbol{v}}{\partial \mathbf{f}}\right) \cdot \mathrm{d} \boldsymbol{p} .
$$

Note the parallelism between the definitions of $\mathbf{p}$ and $\hat{\mathbf{e}}$ in (20) and (29), respectively.

\subsection{Hamiltonian Properties and Bond Graphs for Momentum Equations}

Continuing with the momentum form of the equations, let us first assume the system is holonomic, so that $\mathbf{f}=\dot{\mathbf{q}}$. In this case the derivative of a partial velocity vector can be written as

$$
\frac{\mathrm{d}}{\mathrm{d} t}\left(\frac{\partial \boldsymbol{v}}{\partial \dot{\mathbf{q}}}\right)=\left(\frac{\partial \boldsymbol{v}}{\partial \mathbf{q}}\right)
$$

which is the well-known "cancellation of dots" identity. Therefore the generalized gyration force in (29) reduces to

$$
\hat{\mathbf{e}}=\int\left(\frac{\partial \boldsymbol{v}}{\partial \mathbf{q}}\right) \cdot \mathrm{d} \boldsymbol{p}=\int\left(\frac{\partial \boldsymbol{v}}{\partial \mathbf{q}}\right) \cdot \boldsymbol{v} \mathrm{d} m=\frac{\partial T^{*}}{\partial \mathbf{q}},
$$

where $T^{*}$ is the kinetic co-energy function defined in (18). To get this into Hamiltonian form, we need to convert the kinetic co-energy function $T^{*}$ to the kinetic energy function $T$. Using (26) with $\mathbf{f}=\dot{\mathbf{q}}$, we find

$$
T^{*}(\mathbf{q}, \dot{\mathbf{q}})=T(\mathbf{q}, \mathbf{p}) \equiv(1 / 2) \mathbf{p}^{\top} \mathbf{A}^{-1} \mathbf{p}
$$




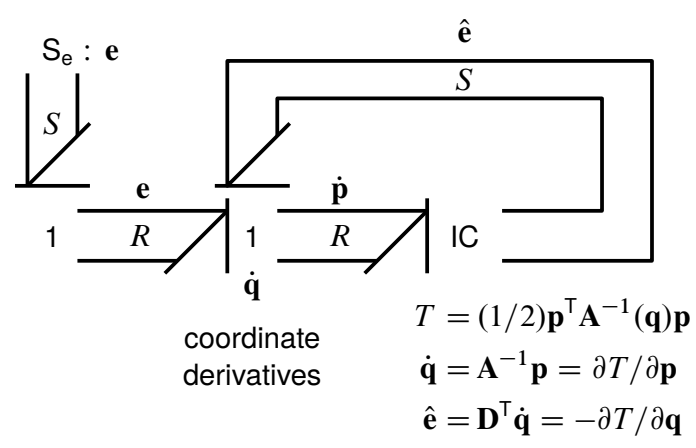

Figure 3. Multibond graph of reduced Kane's equations, holonomic momentum form

Making use of the identity

$$
\frac{\partial \mathbf{A}^{-1}}{\partial q_{r}}=-\mathbf{A}^{-1} \frac{\partial \mathbf{A}}{\partial q_{r}} \mathbf{A}^{-1}
$$

we find we may write the generalized gyration forces as

$$
\hat{\mathbf{e}}=-\frac{\partial T}{\partial \mathbf{q}} .
$$

By partial differentiation of $T$ with respect to $\mathbf{p}$, we also find

$$
\frac{\partial T}{\partial \mathbf{p}}=\mathbf{A}^{-1} \mathbf{p}=\dot{\mathbf{q}}
$$

so that the holonomic momentum equations may be expressed as the Hamiltonian pair

$$
\dot{\mathbf{p}}=\mathbf{e}-\frac{\partial T}{\partial \mathbf{q}}, \quad \dot{\mathbf{q}}=\frac{\partial T}{\partial \mathbf{p}} .
$$

We regard the holonomic momentum equations as fully Hamiltonian, because the right side of both equations is generated by partial differentiations of the kinetic energy function $T$ (we are ignoring the generalized impressed force $\mathbf{e}$ here, as is conventional). In this case we say that the kinetic energy function $T$ is monogenic [16, p. 30].

To develop a bond graph for the holonomic momentum form, we differentiate (33):

$$
\begin{aligned}
\frac{\mathrm{d} T}{\mathrm{~d} t} & =\frac{\partial T}{\partial \mathbf{p}^{\top}} \dot{\mathbf{p}}+\frac{\partial T}{\partial \mathbf{q}^{\top}} \dot{\mathbf{q}} \\
& =\mathbf{p}^{\top} \mathbf{A}^{-1} \dot{\mathbf{p}}-(1 / 2) \mathbf{p}^{\top} \mathbf{A}^{-1} \dot{\mathbf{A}} \mathbf{A}^{-1} \mathbf{p} \\
& =\dot{\mathbf{q}}^{\top}(\dot{\mathbf{p}})-\dot{\mathbf{q}}^{\top}\left(\mathbf{D}^{\top} \dot{\mathbf{q}}\right),
\end{aligned}
$$

which again suggests a two-port kinetic energy store, with effort $\dot{\mathbf{p}}$ on one port, and effort $\mathbf{D}^{\top} \dot{\mathbf{q}}$ on the other. The resulting bond graph of the holonomic momentum form of Kane's equations in shown in Fig. 3. In this case, the required kinetic energy $(T)$ store is identified as the standard multibond IC element, first used (as a field rather than a multibond element) specifically for this purpose by Karnopp in [17]. The multibond IC element is defined in such a way that it requires the monogenic property of $T$ to hold, i.e. the flow on the (left) I-port and the effort on the (right) C-port are given by the Hamiltonian differentiation operations on $T$ defined above, and shown in the figure.

Now for the nonholonomic case, we can still differentiate $T$ with respect to $\mathbf{p}$, and get the valid equation $\mathbf{f}=\partial T / \partial \mathbf{p}=\mathbf{A}^{-1} \mathbf{p}$. However, $\hat{\mathbf{e}}$ is no longer generated by $\partial T / \partial \mathbf{q}$, so that the right side of only one of the pair is generated by $T$ :

$$
\dot{\mathbf{p}}=\mathbf{e}+\hat{\mathbf{e}}, \mathbf{f}=\frac{\partial T}{\partial \mathbf{p}} .
$$




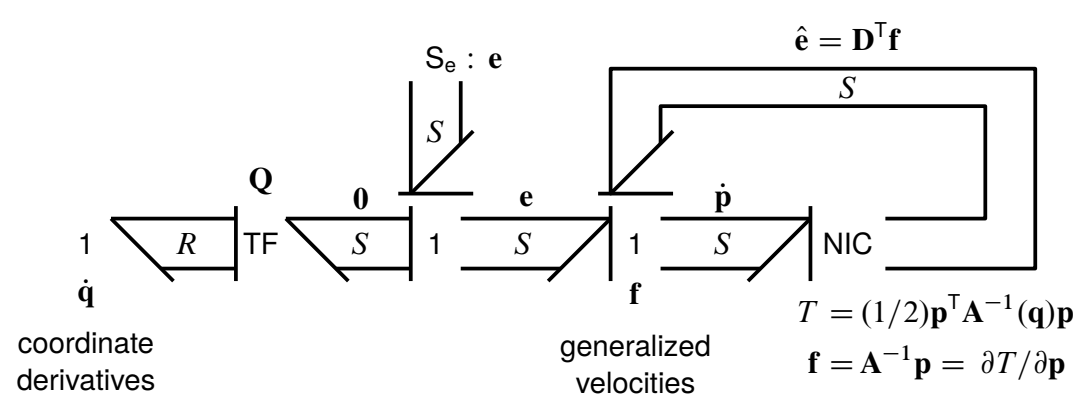

Figure 4. Multibond graph of reduced Kane's equations, nonholonomic momentum form

Thus, in the nonholonomic case, the kinetic energy function $T$ is not monogenic, because it doesn't generate the right sides of both equations in the pair. We may however regard the nonholonomic momentum equations as being partially Hamiltonian, because the kinetic energy function generates one of the pair. A bond graph for this case is shown in Fig. 4; in this case we show the required $T$ store as a newly defined bond-graph element, the nonholonomic IC, or NIC element. This energy store has the partially Hamiltonian property defined above, which generates the flow $\mathbf{f}$ on the left (I) port from $\partial T / \partial \mathbf{p}$, but requires the $\mathbf{D}^{\top}$ matrix to generate the effort $\hat{\mathbf{e}}$ on the right $(\mathrm{C})$ port. Importantly, when the system being described is actually holonomic, the NIC element reduces to the standard IC element defined above.

\subsection{Lagrangian Form and Bond Graphs for Velocity Equations}

An alternative form of the velocity equations in Sec. 3.4 can be developed from the momentum form developed in Secs. 3.5-3.6, which is partially Lagrangian in the nonholonomic case, and fully Lagrangian in the holonomic case. Starting from the generalized momentum function $\hat{\mathbf{p}}(\mathbf{q}, \mathbf{f})$ defined above in Eq. (22), we take the total derivative of $\hat{\mathbf{p}}(\mathbf{q}, \mathbf{f})$ symbolically, finding

$$
\dot{\hat{\mathbf{p}}}=\left(\frac{\partial \hat{\mathbf{p}}}{\partial \mathbf{f}^{\top}}\right) \dot{\mathbf{f}}+\left(\frac{\partial \hat{\mathbf{p}}}{\partial \mathbf{q}^{\top}}\right) \dot{\mathbf{q}}
$$

Then using the definition of $\hat{\mathbf{p}}$ in (22), we see that $\partial \hat{\mathbf{p}} / \partial \mathbf{f}^{\top}$ is equal to $\mathbf{A}$, so we have

$$
\dot{\hat{\mathbf{p}}}=\mathbf{A} \dot{\mathbf{f}}+\left(\frac{\partial \hat{\mathbf{p}}}{\partial \mathbf{q}^{\top}}\right) \dot{\mathbf{q}}
$$

Substituting this into the momentum equation of motion (28), we have

$$
\mathbf{A} \dot{\mathbf{f}}+\left(\frac{\partial \hat{\mathbf{p}}}{\partial \mathbf{q}^{\top}}\right) \dot{\mathbf{q}}=\mathbf{e}+\hat{\mathbf{e}}
$$

This form of the nonholonomic velocity equations is believed to be a new result, which is useful in several ways. We call it the partially Lagrangian form of the velocity equations.

First, it has a partially Lagrangian property, as we shall explain. The left side of (41) is generated by the left side of (40), which in turn is generated by differentiation of the kinetic co-energy function, i.e.

$$
\dot{\hat{\mathbf{p}}}=\frac{\mathrm{d}}{\mathrm{d} t}\left(\frac{\partial T^{*}}{\partial \mathbf{f}}\right)
$$

where $T^{*}$ is the nonholonomic kinetic co-energy function defined in (18). Therefore we can write (41) in the form

$$
\frac{\mathrm{d}}{\mathrm{d} t}\left(\frac{\partial T^{*}}{\partial \mathbf{f}}\right)=\mathbf{e}+\hat{\mathbf{e}}
$$




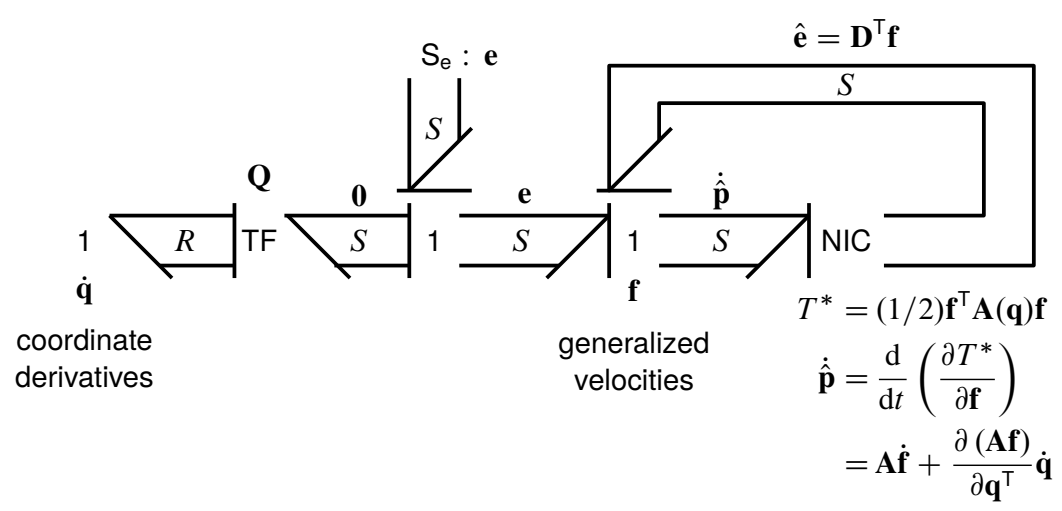

Figure 5. Multibond graph of reduced Kane's equations, nonholonomic partially Lagrangian velocity form

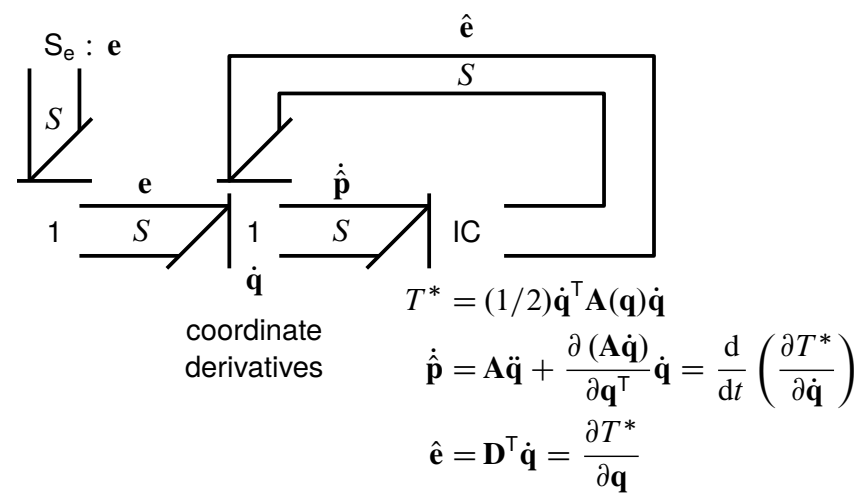

Figure 6. Multibond graph of reduced Kane's equations, holonomic Lagrangian form

Figure 5 displays a multibond graph of the partially Lagrangian form of the velocity equations, using the NIC multibond-graph element defined in Sec. 3.6. As in the momentum case, the energy function $T^{*}$ associated with the NIC element generates the column matrix of efforts on the left (I) side of the element $(\dot{\hat{\mathbf{p}}})$, but not the column matrix of efforts $\hat{\mathbf{e}}$ on the right (C) side of the element.

If the system were fully holonomic, the left side of Eq. (42) would be $\mathrm{d}\left(\partial T^{*} / \partial \dot{\mathbf{q}}\right) / \mathrm{d} t$, while, using (32), the right side would be $e+\partial T^{*} / \partial \mathbf{q}$. Clearly, these are are Lagrange's equations. Therefore Eq. (42) demonstrates a partially Lagrangian property, which becomes completely Lagrangian in case that the system is holonomic. From another point of view, in the holonomic case, the kinetic co-energy function $T^{*}$ is seen to be monogenic, in that it generates both sides of the equation (setting $\mathbf{e}$ aside), while in the nonholonomic case, it generates only one side of the equation. Thus, as in the momentum equations, the associated energy function is or is not monogenic according as the system is or is not holonomic. Figure 6 displays a multibond graph of the velocity equations in the fully Lagrangian form, using again the standard multibond-graph IC element.

Besides having the partially Lagrangian property, Eq. (41) provides a useful alternative implementation for the computing the product Df. Comparing (41) to (14), we find

$$
\mathbf{D f}=\frac{\partial \hat{\mathbf{p}}}{\partial \mathbf{q}^{\top}} \dot{\mathbf{q}}-\hat{\mathbf{e}} .
$$

The expression on the right side of (43) may be simpler to compute by analysis than the one on the left side, particularly if one or more of the coordinates $\mathbf{q}$ does not appear in the expression for $\hat{\mathbf{p}}$. A 
generalized coordinate $q_{r}$ is defined as ignorable if it does not appear explicitly in the momentum function $\hat{\mathbf{p}}$ (or equivalently the mass matrix $\mathbf{A}$ ). If all generalized coordinates are ignorable, then

$$
\begin{gathered}
\mathbf{D f}=-\hat{\mathbf{e}}=-\mathbf{D}^{\top} \mathbf{f} . \\
\text { (all coordinates ignorable) }
\end{gathered}
$$

This could also be derived directly from Eq. 17.

\subsection{Extension to Rheonomic Systems, Including Generalized Constraint Forces.}

Now, in the final stage of our analysis, we complete the method of constraint relaxation, by enforcing the previously relaxed $S_{\mathrm{c}}$ rheonomic flow constraints, thereby removing the limitation of the analysis to scleronomic systems, and allowing the determination of the reactions associated with the rheonomic constraints. Therefore in addition to partitioning the flow matrix $\mathbf{f}$ according to (2), we must partition the effort matrix $\mathbf{e}$ as

$$
\mathbf{e}=\left[\begin{array}{c}
\mathrm{u} \\
{ }^{\mathrm{c}} \\
{ }^{\mathrm{e}}
\end{array}\right]
$$

where ${ }^{\mathrm{u}} \mathbf{e}$ is the column vector of impressed efforts corresponding to ${ }^{\mathrm{u}} \mathbf{f}$, and ${ }^{\mathrm{c}} \mathbf{e}$ is the column matrix of constraint reactions to be found, corresponding to the directly known flow constraints ${ }^{\mathrm{c}} \mathbf{f}$.

Equation (14), the reduced velocity form of the equations of motion, when partitioned according to this scheme, becomes

$$
\left[\begin{array}{c}
{ }^{\mathrm{u}} \mathbf{e} \\
{ }^{\mathrm{c}} \mathbf{e}
\end{array}\right]=\left[\begin{array}{cc}
{ }^{\mathrm{u}} \mathbf{A} & { }^{\mathrm{uc}} \mathbf{A} \\
{ }^{\mathrm{c}} \mathbf{A} & { }^{\mathrm{cc}} \mathbf{A}
\end{array}\right]\left[\begin{array}{c}
{ }^{\mathrm{u}} \mathbf{f} \\
{ }^{{ }^{\mathrm{f}}} \mathbf{\mathbf { f }}
\end{array}\right]+\left[\begin{array}{c}
{ }^{\mathrm{u}} \mathbf{D} \\
{ }^{\mathrm{c}} \mathbf{D}
\end{array}\right] \mathbf{f},
$$

where we are partitioning $\mathbf{A}$ according to its rows and columns, but we are partitioning $\mathbf{D}$ only according to its rows. The first row of (46) gives a solution for ${ }^{\mathrm{u}} \dot{\mathbf{f}}$ as

$$
{ }^{\mathrm{u}} \dot{\mathbf{f}}=\left[{ }^{\mathrm{uu}} \mathbf{A}\right]^{-1}\left[{ }^{\mathrm{u}} \mathbf{e}-{ }^{\mathrm{uc}} \mathbf{A}^{\mathrm{c}} \dot{\mathbf{f}}-{ }^{\mathrm{u}} \mathbf{D} \mathbf{f}\right],
$$

while the second row directly gives the solution for the constraint reactions as

$$
{ }^{\mathrm{c}} \mathbf{e}={ }^{\mathrm{cu}} \mathbf{A}{ }^{\mathrm{u}} \dot{\mathbf{f}}+{ }^{\mathrm{cc}} \mathbf{A}{ }^{\mathrm{c}} \dot{\mathbf{f}}+{ }^{\mathrm{c}} \mathbf{D} \mathbf{f}
$$

in order to implement these computationally (47) must be evaluated before (48).

To work with the reduced momentum form of the equations of motion, we may proceed similarly. The first row of the partitioned form of (25) gives an equation for ${ }^{\mathrm{u}} \dot{\mathbf{p}}$, as

$$
{ }^{\mathrm{u}} \dot{\mathbf{p}}={ }^{\mathrm{u}} \mathbf{e}+{ }^{\mathrm{u}}\left[\mathbf{D}^{\top}\right] \mathbf{f},
$$

while the first row of the partitioned form of (21) yields the solution for ${ }^{\mathrm{u}} \mathbf{f}$ as

$$
{ }^{\mathrm{u}} \mathbf{f}=\left[{ }^{\mathrm{uu}} \mathbf{A}\right]^{-1}\left[{ }^{\mathrm{u}} \mathbf{p}-{ }^{\mathrm{uc}} \mathbf{A}^{\mathrm{c}} \mathbf{f}\right] .
$$

The momentum formulation for the unconstrained flows, $(49,50)$, shows a clear computational advantage over the velocity formulation $(47,48)$ in that the known constrained flows do not have to be differentiated to find ${ }^{\mathrm{u}} \mathbf{f}$ in the momentum formulation, whereas they do in the velocity formulation. However, it is not possible to avoid differentiating the constrained flows in order to find the constraint reactions ${ }^{c} \mathbf{e}$. Although a momentum formulation for the constraint reactions can be developed [18], it offers no computational advantages over the velocity formulation, and therefore the velocity formulation for the constraint reactions, (48), is recommended for use in all cases. 


\section{RIGID-BODY FORMS OF THE SYSTEM MATRICES}

We consider now a system of $N$ rigid bodies indexed by $n$, with body $n$ having angular velocity $\omega_{n}$ in the inertial frame, and its center of mass having linear velocity $\boldsymbol{v}_{n}$ in the same frame. Body $n$ will have total mass $m_{n}$, and rotational inertia tensor $\mathbf{I}_{n}$. The velocity vectors for each body can be written as

$$
\boldsymbol{\omega}_{n}=\left(\frac{\partial \boldsymbol{\omega}_{n}}{\partial \mathbf{f}^{\top}}\right) \mathbf{f}, \quad \boldsymbol{v}_{n}=\left(\frac{\partial \boldsymbol{v}_{n}}{\partial \mathbf{f}^{\top}}\right) \mathbf{f} .
$$

Euler defined the linear and angular momentum vectors as

$$
\boldsymbol{p}_{n}=m_{n} \boldsymbol{v}_{n}, \quad \boldsymbol{h}_{n}=\mathbf{I}_{n} \cdot \boldsymbol{\omega}_{n},
$$

respectively. We assume that the total impressed force acting through the center of mass is $\boldsymbol{F}_{n}$, and the total impressed moment acting about the center of mass is $\boldsymbol{M}_{n}$.

By Stieltjes integration of the particle contributions over each body, we may show the following system relationships, which are listed without proof (using the summation convention over $n$ ):

$$
\begin{gathered}
\mathbf{e}=\left(\frac{\partial \boldsymbol{v}_{n}}{\partial \mathbf{f}}\right) \cdot \boldsymbol{F}_{n}+\left(\frac{\partial \boldsymbol{\omega}_{n}}{\partial \mathbf{f}}\right) \cdot \boldsymbol{M}_{n} \\
\mathbf{p}=\hat{\mathbf{p}}(\mathbf{q}, \mathbf{f})=\left(\frac{\partial \boldsymbol{v}_{n}}{\partial \mathbf{f}}\right) \cdot \boldsymbol{p}_{n}+\left(\frac{\partial \boldsymbol{\omega}_{n}}{\partial \mathbf{f}}\right) \cdot \boldsymbol{h}_{n} \\
\mathbf{A}=\frac{\partial \hat{\mathbf{p}}}{\partial \mathbf{f}^{\top}}=m_{n}\left(\frac{\partial \boldsymbol{v}_{n}}{\partial \mathbf{f}}\right) \cdot\left(\frac{\partial \boldsymbol{v}_{n}}{\partial \mathbf{f}^{\top}}\right)+\left(\frac{\partial \boldsymbol{\omega}_{n}}{\partial \mathbf{f}}\right) \cdot \mathbf{I}_{n} \cdot\left(\frac{\partial \boldsymbol{\omega}_{n}}{\partial \mathbf{f}^{\top}}\right) \\
\mathbf{D}=m_{n}\left(\frac{\partial \boldsymbol{v}_{n}}{\partial \mathbf{f}}\right) \cdot \frac{\mathrm{d}}{\mathrm{d} t}\left(\frac{\partial \boldsymbol{v}_{n}}{\partial \mathbf{f}^{\top}}\right)+\left(\frac{\partial \boldsymbol{\omega}_{n}}{\partial \mathbf{f}}\right) \cdot \mathbf{I}_{n} \cdot \frac{\mathrm{d}}{\mathrm{d} t}\left(\frac{\partial \boldsymbol{\omega}_{n}}{\partial \mathbf{f}^{\top}}\right)+\left(\frac{\partial \boldsymbol{\omega}_{n}}{\partial \mathbf{f}}\right) \cdot\left(\boldsymbol{\omega}_{n} \times \frac{\partial \boldsymbol{h}_{n}}{\partial \mathbf{f}^{\top}}\right) \\
\hat{\mathbf{e}}=\mathbf{D}^{\top} \mathbf{f}=\frac{\mathrm{d}}{\mathrm{d} t}\left(\frac{\partial \boldsymbol{v}_{n}}{\partial \mathbf{f}}\right) \cdot \boldsymbol{p}_{n}+\frac{\mathrm{d}}{\mathrm{d} t}\left(\frac{\partial \boldsymbol{\omega}_{n}}{\partial \mathbf{f}}\right) \cdot \boldsymbol{h}_{n} .
\end{gathered}
$$

All the reduced form equations derived above for particle systems apply equally well for rigid-body systems, when these results for the system matrices are used in the equations.

For holonomic systems, we can use the previously mentioned "cancellation of dots" identity for the linear velocity vectors, as well as an additional identity for the angular velocity vectors, which is

$$
\frac{\mathrm{d}}{\mathrm{d} t}\left(\frac{\partial \boldsymbol{\omega}_{n}}{\partial \dot{\mathbf{q}}}\right)=\left(\frac{\partial \boldsymbol{\omega}_{n}}{\partial \mathbf{q}}\right)+\omega_{n} \times\left(\frac{\partial \omega_{n}}{\partial \dot{\mathbf{q}}}\right) .
$$

Under these circumstances, we have the following simplifications:

$$
\begin{gathered}
\mathbf{D}=m_{n}\left(\frac{\partial \boldsymbol{v}_{n}}{\partial \dot{\mathbf{q}}}\right) \cdot\left(\frac{\partial \boldsymbol{v}_{n}}{\partial \mathbf{q}^{\top}}\right)+\left(\frac{\partial \boldsymbol{\omega}_{n}}{\partial \dot{\mathbf{q}}}\right) \cdot \boldsymbol{I}_{n} \cdot\left(\frac{\partial \boldsymbol{\omega}_{n}}{\partial \mathbf{q}^{\top}}\right), \\
\hat{\mathbf{e}}=\mathbf{D}^{\top} \dot{\mathbf{q}}=\left(\frac{\partial \boldsymbol{v}_{n}}{\partial \mathbf{q}}\right) \cdot \boldsymbol{p}_{n}+\left(\frac{\partial \boldsymbol{\omega}_{n}}{\partial \mathbf{q}}\right) \cdot \boldsymbol{h}_{n} .
\end{gathered}
$$

\subsection{Relationship to Previous Results}

In the authors' previously published paper [3], which covers only the scleronomic momentum form for rigid-body systems, we define the $\mathbf{C}$ matrix as

$$
\mathbf{C}=m_{n} \frac{\mathrm{d}}{\mathrm{d} t}\left(\frac{\partial \boldsymbol{v}_{n}}{\partial \mathbf{f}}\right) \cdot\left(\frac{\partial \boldsymbol{v}_{n}}{\partial \mathbf{f}^{\top}}\right)+\frac{\mathrm{d}}{\mathrm{d} t}\left(\frac{\partial \boldsymbol{\omega}_{n}}{\partial \mathbf{f}}\right) \cdot \mathbf{I}_{n} \cdot\left(\frac{\partial \boldsymbol{\omega}_{n}}{\partial \mathbf{f}^{\top}}\right)
$$

from which it is clear that $\mathbf{D}$ and $\mathbf{C}$ are related according to

$$
\mathbf{D}=\mathbf{C}^{\boldsymbol{\top}}+\left(\frac{\partial \boldsymbol{\omega}_{n}}{\partial \mathbf{f}}\right) \cdot\left(\boldsymbol{\omega}_{n} \times \frac{\partial \boldsymbol{h}_{n}}{\partial \mathbf{f}^{\top}}\right) .
$$

The $\mathbf{A}$ and $\mathbf{C}$ matrices are sufficient to define the momentum forms, without requiring the $\mathbf{D}$ matrix. 


\section{REFERENCES}

[1] Paynter, H.: Analysis and design of engineering systems; class notes for MIT course 2.751 (1961)

[2] Karnopp, D., Margolis, D., Rosenberg, R.: System Dynamics. Third edn. Wiley (2000)

[3] Phillips, J.R., Amirouche, F.: A momentum form of Kane's equations for scleronomic systems. Mathematical and Computer Modelling of Dynamical Systems 24(2) (2018) 143-169

[4] Kane, T.R.: Dynamics of nonholonomic systems. J. Appl. Mech. 28(4) (1961) 574-578

[5] Kane, T.R., Wang, C.F.: On the derivation of equations of motion. J. Soc. Indust. Appl. Math. 13(2) (1965) 487-492

[6] Kane, T.R., Levinson, D.A.: Dynamics: Theory and Applications. McGraw-Hill (1985)

[7] Amirouche, F.: Fundamentals of multibody dynamics: theory and applications. Springer Science \& Business Media (2007)

[8] Kane, T., Banerjee, A.: A conservation theorem for simple nonholonomic systems. Journal of Applied Mechanics 50(3) (1983) 647-651

[9] Banerjee, A.K.: Order-n formulation of equations of motion with efficient choices of motion variables. Guidance, Navigation, and Control and Co-located Conferences. American Institute of Aeronautics and Astronautics (August 1994)

[10] Mitiguy, P.C., Kane, T.R.: Motion variables leading to efficient equations of motion. The International Journal of Robotics Research 15(5) (1996) 522-532

[11] Roithmayr, C.M., Hodges, D.H.: Dynamics: Theory and Application of Kane's Method. Cambridge University Press (2016)

[12] Papastavridis, J.G.: Analytical Mechanics. World Scientific Publishing Co Pte Ltd, Singapore (2014)

[13] Wang, L., Pao, Y.: Jourdain's variational equation and Appell's equation of motion for nonholonomic dynamical systems. Am. J. Phys 71(1) (January 2003)

[14] Breedveld, P.: Multibond graph elements in physical systems theory. J. Franklin Inst. 319(1/2) (1985) 1-36

[15] Fahrenthold, E., Wargo, J.: Vector and tensor based bond graphs for physical systems modeling. Journal of the Franklin Institute 328(5-6) (1991) 833-853

[16] Lanczos, C.: The variational principles of mechanics. University of Toronto press (1970)

[17] Karnopp, D.: An approach to derivative causality in bond graph models of mechanical systems. J. Franklin Inst. 329(1) (1992) 65-75

[18] Phillips, J.R., Amirouche, F.: Corrigendum: a momentum form of Kane's equations for scleronomic systems. Mathematical and Computer Modelling of Dynamical Systems (2018) $1-4$ 